Irish Math. Soc. Bulletin

Number 74, Winter 2014, 25-25

ISSN 0791-5578

\title{
CONFORMAL FIELD THEORIES ON RIEMANN SURFACES OF GENUS $g \geq 1$
}

\author{
MARIANNE LEITNER
}

This is an abstract of the PhD thesis CFTs on Riemann Surfaces of genus $g \geq 1$ in mathematics, written by Dr. Marianne Leitner under the supervision of Prof. Dmitri Zaitsev at the School of Mathematics, TCD, and submitted in August 2013.

The purpose of this thesis is to argue that $N$-point functions of holomorphic fields in rational conformal field theories can be calculated by methods from algebraic geometry. We establish explicit formulae for the 2-point function of the Virasoro field on hyperelliptic Riemann surfaces of genus $g \geq 1$. $N$-point functions for higher $N$ are obtained inductively, and we show that they have a nice graphical representation. We discuss the Virasoro 3-point function with application to the Virasoro $(2,5)$ minimal model.

The formulae involve a finite number of parameters, notably the 0-point function and the Virasoro 1-point function, which depend on the moduli of the surface and can be calculated by differential equations. We propose an algebraic geometric approach that applies to any hyperelliptic Riemann surface. Our discussion includes a demonstration of our methods to the case $g=1$.

SCHOOL OF THEORETICAL PHYSICS, DUBLIN INSTITUTE FOR ADVANCED STUDIES leitner@stp.dias.ie

School of Theoretical Physics, DiAs, Burlington Road, Dublin 4

E-mail address: m.leitner@dias.ie

2010 Mathematics Subject Classification. 00X00, 00X00.

Key words and phrases. Field Theories, Riemann Surface.

Received on 28-3-2014.

Support from Science Foundation Ireland through the Research Frontiers Programme is gratefully acknowledged. 